\title{
188. On the Electronic Analog Computer for Flood Routing
}

\author{
By Tojiro IsHIHARA, Shoitiro HAYAMI, and Shigenori HAYASHI \\ Disaster Prevention Research Institute, Kyoto University \\ (Comm. by Y. TANAKA, M.J.A., Nov. 12, 1954)
}

Japan is confronted with a grave menace of floods, the damages by them amounting to several hundred million dollars every year. It is, now, our urgent problem to establish an economic and efficient flood control project. To achieve this end, we must know, among others, rainfall conditions over catchment basins, topographical and geological features of them, nature of river flow at flood and demands of water utilization. Especially, the flows in natural river channels among them are very complex phenomena; the boundary conditions are incessantly affected by dams, retention pools, tides at estuaries and so on; irregularities of channels and fluctuations of sediment supplies cast their shadows upon the nature of flows. It is almost impossible to find a complete solution of it under such complicated conditions.

According to the Hayami's theory of flood waves [1], however, the basic differential equation of them becomes, approximately,

$$
\frac{\partial H}{\partial t}+A \frac{\partial H^{3 / 2}}{\partial x}=\mu \frac{\partial^{2} H}{\partial x^{2}},
$$

where, $H$ : the water depth, $t:$ the time, $x$ : the distance, and $A$ is a constant determined by the hydraulic resistance and the bottom slope of channel and $\mu$ a numerical constant defined by the various irregularities of river reach and the fluctuations of bottom sediment. Recently, an electronic analog computer based on Muskingum flood routing method [2] has been developed and greatly reduced troubles of computation. But there are inherent defects in the Muskingum principle. In order to improve these deficiencies, the authors have designed and constructed a new type of electronic analog computer based on Eq. (1).

\section{Correspondence of Hydraulic System to Electric System}

Introducing a quantity $Q$ which means a true discharge per unit width defined by

$$
-\frac{\partial Q}{\partial x}=\frac{\partial H}{\partial t},
$$

we get from Eq. (1) 


$$
-\frac{\partial H}{\partial x}=\frac{1}{\mu} Q-\frac{A}{\mu} H^{3 / 2} .
$$

And assuming, as is usually done, the correspondence of hydraulic system to electric one as follows:

$$
\begin{aligned}
& H(\text { water depth })=\alpha v \quad \text { (voltage), } \\
& Q \text { (discharge) }=\theta i \text { (electric current), } \\
& t_{h y} \text { (time) }=\beta t_{e l} \text { (time), } \\
& x_{h y} \text { (distance) }=\gamma x_{e t} \text { (distance), }
\end{aligned}
$$

where $\alpha, \beta, \gamma$ and $\theta$ are coefficients of transformation, Eqs. (2) and (3) may be written in the electric system, respectively,

$$
\begin{gathered}
-\frac{\partial i}{\partial x_{e l}}=\frac{\alpha \gamma}{\theta \beta} \cdot \frac{\partial v}{\partial t_{e l}} \\
-\frac{\partial v}{\partial x_{e l}}=\frac{\theta \gamma}{\mu \alpha} i-\frac{A \alpha^{1 / 2} \gamma}{\mu} v^{3 / 2} .
\end{gathered}
$$

Here, putting,

$$
C=\frac{\alpha \gamma}{\theta \beta}, \quad R=\frac{\theta \gamma}{\mu \alpha} \quad \text { and } \quad k=\frac{A \alpha^{\mathrm{t} / 2} \gamma}{\mu},
$$

where $C$ : the static capacity, $R$ : the electric resistance and $k:$ a certain constant, Eq. (5) becomes

$$
\begin{gathered}
-\frac{\partial i}{\partial x_{e l}}=C \frac{\partial v}{\partial t_{e l}} \\
-\frac{\partial v}{\partial x_{e l}}=R i-k v^{3 / 2}
\end{gathered}
$$

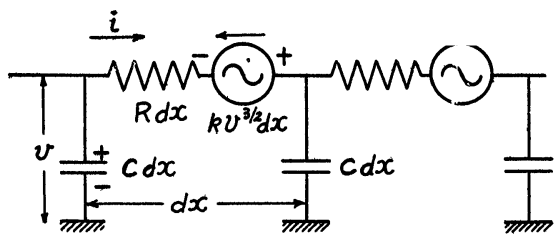

Fig. 1. Schematic circuit diagram represented by Eqs. (7) and (8)

These are partial differential equations of a cable with series resistance $R$, shunt capacity $C$ and non-linear distributed electro-motive force $k v^{3 / 2}$ in series arm, per unit length as shown in Fig. 1.

\section{Circuit of Computer}

The most important problem is how to design the series electromotive force $k v^{3 / 2}$. After comparison of various methods, we have adopted the following method to realize the non-linear element.

Assuming $i_{0}=k v^{3 / 2} / R=k^{\prime} v^{3 / 2}$, we get from Eq. (8)

$$
-\frac{\partial v}{\partial x_{e l}}=R\left(i-i_{0}\right) \text {. }
$$

The circuit shown in Fig. 2 represents what is shown by Eqs. (7) and (9), where the non-linear electro-motive force as shown in 
Fig. 1 is replaced by the non-linear forced current $\ddot{i}_{0}$. This current may be realized by $v_{g}$ (the grid voltage) and $i_{p}$ (the plate current) characteristics of sharp cut-off pentode 6SJ7. That is,

$$
i_{p} \cong k^{\prime \prime} v_{g}^{3 / 2}
$$

where $k^{\prime \prime}$ is a constant defined by the pentode. If the fractional voltage of $v$ is applied to the grid, we find,

$$
i_{p} \cong k^{\prime \prime} a^{3 / 2} v^{3 / 2}
$$

where $a$ is the divider ratio. Adjusting the divider ratio $a$ such as $k^{\prime \prime} a^{3 / 2}=k^{\prime}$, therefore, we may use $i_{p}$ instead of $i_{0}$. A schematic circuit diagram of

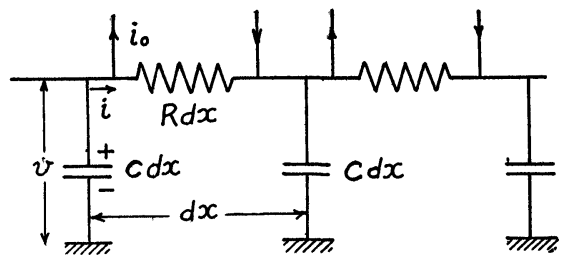

Fig. 2. Schematic circuit diagram represented by Eqs. (7) and (9) the computing river element designed in this manner is shown in Fig. 3. In this figure, $R_{g}$ is added in parallel to $C$ in order to divide the voltage $v$, so that, strictly speaking, Eq. (7) becomes

$$
-\frac{\partial i}{\partial x_{e l}}=C \frac{\partial v}{\partial t_{e l}}+\frac{v}{R_{g}} \text {. }
$$

In practical design, it is necessary to make $R_{g}$ sufficiently large so as to make the term $v / R_{g}$ negligibly small in comparison with the term $C \frac{\partial v}{\partial t_{e l}}$.

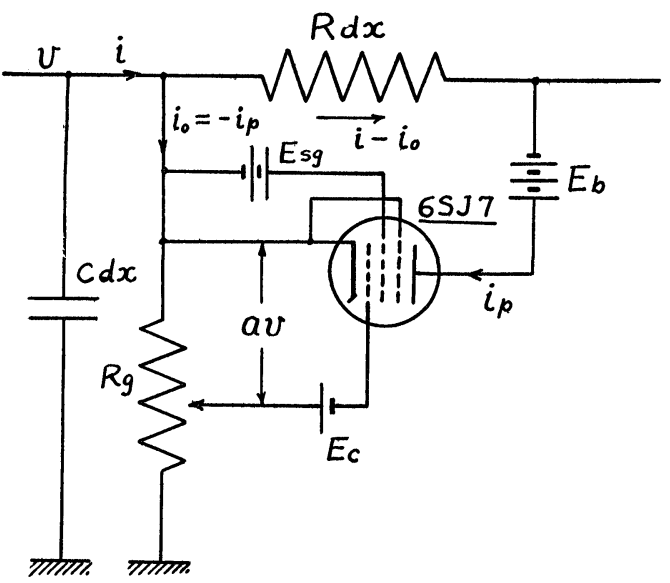

Fig. 3. Schematic circuit diagram of computing river element

\section{Practical Design of Computer for the River Kiso}

For practical application, an example of the reach from Kasamatsu to Naruto in the River Kiso will be taken. The length of this reach is $16.05 \mathrm{~km}$ and its bottom slope is $S=2.213 \times 10^{-4}$. The value of $A=C^{\prime} \sqrt{S}$ becomes $0.3 \sim 0.6$ against the value of Chézy's coefficient for mean velocity $C^{\prime}=20 \sim 40$ and $\mu$ is assumed $10^{2} \sim 10^{4}$, where all values are shown in metre-second unit.

For practical purpose, it is preferable to set up an electronic analog computer operating with slow speed and analogize this reach with four meshes of concentrated electric circuits. If we assume that one day in hydraulic system corresponds to 2.4 sec in electric 
[Vol. 30,

one and one metre in water stage to ten volts, we get

$$
\alpha=10^{-1}, \beta=3.6 \times 10^{4} \text { and } \gamma=4.0 \times 10^{3} \text {, }
$$

using $\mu=10^{3}$ and $A=0.4$ as average values of them. The results obtained by the above-mentioned relations are summarized in Table I.

Table I

The relation of analogue of two systems

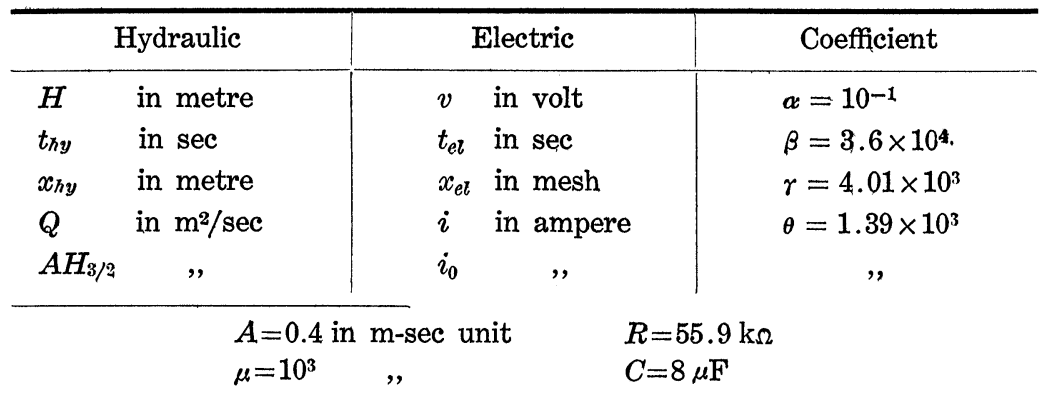

The coefficients $A$ and $\mu$ in Eq. (1) are different for each river and for every reach of a river. It is necessary to be able to change easily these values in the authors' computer. If the value of $C$ remains constant, the values of $A$ and $\mu$ depend on the divider ratio $a$ and the resistance $R$, respectively. Therefore, $a$ and $R$ should be adjustable within actual range of values of $A$ and $\mu$ by use of variable resistors.

The recorder is an ink-writing oscillograph with specially designed D.C. amplifier not so as to disturb the condition of computing circuit. The input table consists of many copper plates insulated by bakelite sheets inserted between the conductors and a brush which slides on it. Each copper plate being kept to the potential equivalent to the flood water stage at each time, the potential of brush generated by sliding is introduced to the river elements as the input signal.

\section{Solutions of Eq. (1) and Application to Flood Routing}

Fig. 4 shows the solutions of Eq. (1) under the following conditions calculated by the authors' computer.

Initial condition:

Boundary condition:

$$
H=0 \mathrm{~m}, \quad \text { at } t=0
$$

$$
\begin{aligned}
& H=\left\{\begin{array}{l}
0 \mathrm{~m}, t \leqq 0, t>t_{0}, \\
H_{0}, \quad 0<t \leqq t_{0},
\end{array} \quad \text { at } x=0 \mathrm{~m}\right. \\
& H=0 \mathrm{~m}, \quad \text { at } x=40 \mathrm{~km}
\end{aligned}
$$

In Fig. 4 (a), the solutions at $x=26 \mathrm{~km}$, when $H_{0}=6 \mathrm{~m}, t_{0}=2.16 \mathrm{hr}$, $A=0.474$ and the value of $\mu$ is variable, are shown and, in Fig. 4 (b), those at $x=24 \mathrm{~km}$, when $t_{0}=2.14 \mathrm{hr}, A=0.474, \mu=1.12 \times 10^{3}$ and the 
value of $H_{0}$ is variable. It is seen, from these figures, that the solutions are very complex and the effect of nonlinear element appears obviously.

As an example of application to the actual flood routing, the computed result for the River Kiso mentioned above is shown in Fig. 5 which shows good coincidence with the actual hydrograph. In order to route a flood by the authors' computer, the values of $A$ and $\mu$ for the river reach must be known. However, it is unable to estimate them exactly except by means of observations. In practice, a number of records are taken by the computer with various values of $A$ and $\mu$, refering to the

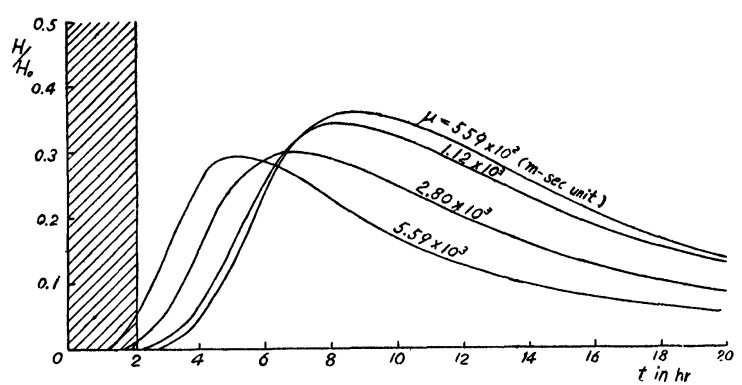

(a) When the value of $\mu$ is variable

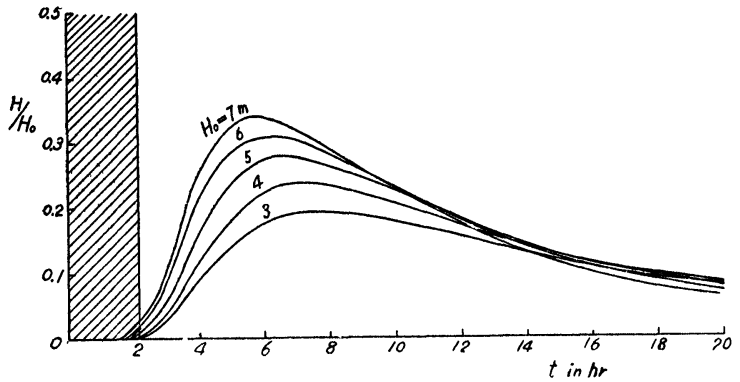

(b) When the wave height $H_{0}$ is variable

Fig. 4. Responses for rectangular wave

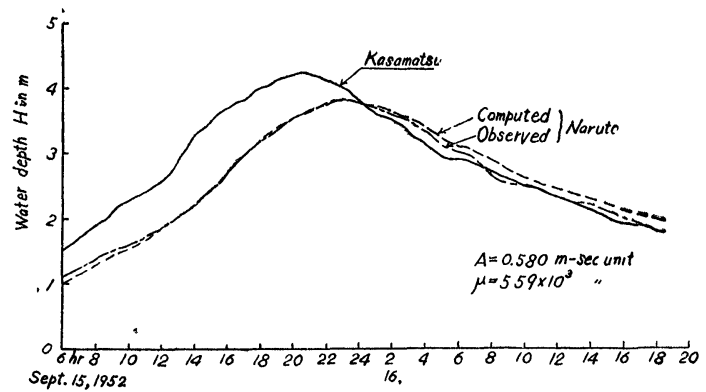

Fig. 5. An example of flood routing past actual records of flood in the river, and the most suitable values of them from these calculated and actual records may be found out. And also, if the secular variations of $A$ and $\mu$ were determined, it might be possible to show quantitatively how the character of a river changes with years.

\section{References}

[1] S. Hayami: On the propagation of flood waves, Disaster Prevention Research Institute, Kyoto University, Bulletin, No. 1, Dec., 1952.

[2] R. K. Linsley, L. W. Foskett, and M. A. Kohler: Use of electrical analogy in flood wave analysis, Assemblée generale d'Oslo 19-28 août 1948, Association Internationale d'Hydrologie Scientifique, $\mathbf{1}$. 
